\title{
Crystal structure of 2-[(3,4-dimethoxy)-phenethyl-3-(3,4-dimethoxy)- phenethylamino]-2,3-dihydro-isoindol-1-one, $\mathrm{C}_{28} \mathrm{H}_{32} \mathrm{~N}_{2} \mathrm{O}_{5}$
}

\author{
E. Sobarzo-Sánchez*,I, B. K. Cassels ${ }^{\mathrm{I}}$, L. Castedo ${ }^{\mathrm{II}}$ and L. Valencia-Matarranz ${ }^{\mathrm{III}}$ \\ I Universidad de Chile, Facultad de Ciencias, Departamento de Química, Casilla 653 Santiago, Chile \\ II Universidad de Santiago de Compostela, Facultad de Química, Departamento de Química Orgánica y Unidad Asociada al C.S.I.C., ES-15706 Santiago de \\ Compostela, Spain \\ III Universidad de Santiago de Compostela, Facultad de Química, Departamento de Química Inorgánica, ES-15782 Santiago de Compostela, Spain
}

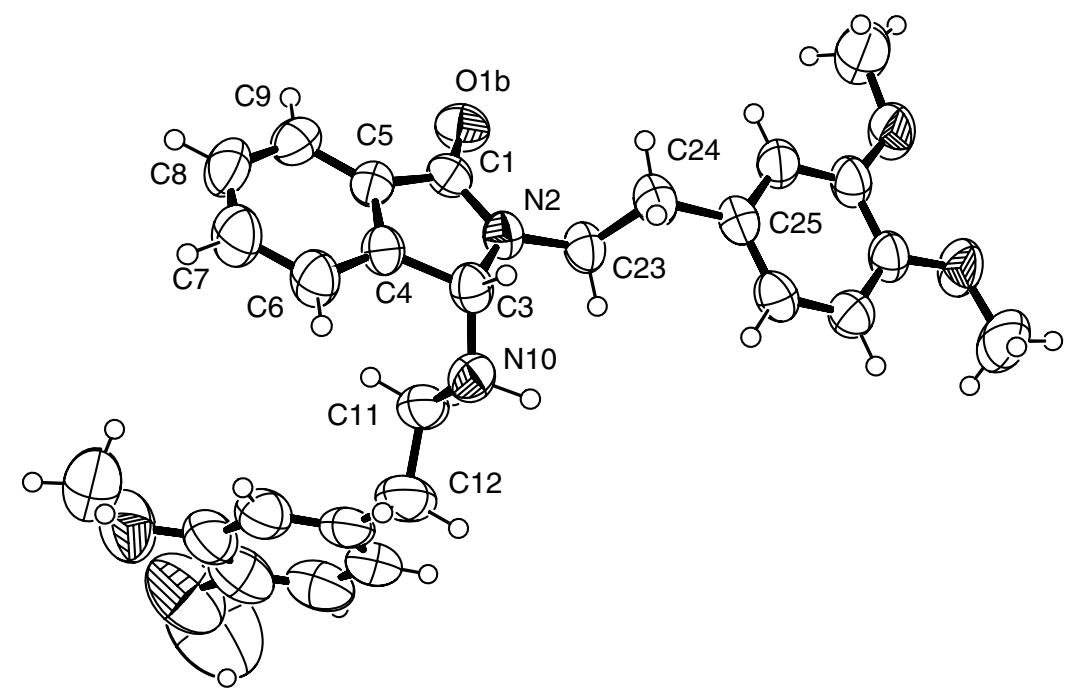

\begin{abstract}
$\mathrm{C}_{28} \mathrm{H}_{32} \mathrm{~N}_{2} \mathrm{O}_{5}$, monoclinic, $P 12{ }_{1} / a 1$ (No. 14), $a=9.5162(6) \AA$,

$b=16.0013(9) \AA, c=17.8001(8) \AA, \beta=102.883(6)^{\circ}$,

$V=2642.2 \AA^{3}, Z=4, R_{\mathrm{gt}}(F)=0.051, w R_{\mathrm{ref}}\left(F^{2}\right)=0.171$,

$T=293 \mathrm{~K}$
\end{abstract}

\section{Source of material}

A solution of phthalaldehydic acid $(1 \mathrm{~g}, 6.67 \mathrm{mmol})$ in $30 \mathrm{~mL}$ of toluene was treated with homoveratrylamine $(2.25 \mathrm{~mL}$, $13.3 \mathrm{mmol}$ ) and refluxed under a Dean-Stark trap with constant stirring for 2 hours. After concentrating to dryness, the title product was purified by crystallization in cyclohexane affording white needles (yield 95\%).

\section{Experimental details}

The hydrogen atom H10B (linked to N10) was located on a difference electron-density map and refined. The other hydrogen atoms were included in idealised positions with isotropic displacement parameters.

\section{Discussion}

The isoindolone moiety is planar and lies parallel to the phenethylamine linked to $\mathrm{N} 2$, having a torsion angle $\mathrm{C} 25-\mathrm{C} 24-\mathrm{C} 23-\mathrm{N} 2$ of $175.4(2)^{\circ}$. All methoxyl group carbon atoms are coplanar with the respective aromatic rings. However, the phenethylamine linked to $\mathrm{C} 3$ is twisted with respect to the isoindolone moiety forming a dihedral angle $\mathrm{C} 3-\mathrm{N} 10-\mathrm{C} 11-\mathrm{C} 12$ of $-161.5(2)^{\circ}$.
Table 1. Data collection and handling.

$\begin{array}{ll}\text { Crystal: } & \text { colourless prism, size } 0.04 \times 0.16 \times 0.4 \mathrm{~mm} \\ \text { Wavelength: } & \mathrm{Cu} K_{\alpha} \text { radiation }(1.5418 \AA) \\ \mu: & 6.67 \mathrm{~cm}^{-1} \\ \text { Diffractometer, scan mode: } & \text { Enraf Nonius CAD4, } \omega / 2 \theta \\ 2 \theta_{\text {max }}: & 145.88^{\circ} \\ N(h k l)_{\text {measured }}, N(h k l)_{\text {unique }}: & 5440,5271 \\ \text { Criterion for } I_{\text {obs }}, N(h k l)_{\mathrm{gt}}: & I_{\mathrm{obs}}>2 \sigma\left(I_{\mathrm{obs}}\right), 2589 \\ N(\text { param })_{\text {refined }} & 325 \\ \text { Programs: } & \text { SIR-97 [1], SHELXL-97 [2], ORTEP-3 [3], } \\ & \text { WinGX [4] }\end{array}$

Table 2. Atomic coordinates and displacement parameters (in $\AA^{2}$ ).

\begin{tabular}{lllrrl}
\hline Atom & Site & $x$ & $y$ & \multicolumn{1}{c}{$z$} & $U_{\text {iso }}$ \\
\hline $\mathrm{H}(3)$ & $4 e$ & 1.0462 & 0.1461 & 0.0404 & 0.066 \\
$\mathrm{H}(6)$ & $4 e$ & 1.0267 & 0.0808 & -0.1198 & 0.083 \\
$\mathrm{H}(7)$ & $4 e$ & 0.8939 & -0.0237 & -0.1913 & 0.097 \\
$\mathrm{H}(8)$ & $4 e$ & 0.6889 & -0.0755 & -0.1598 & 0.092 \\
$\mathrm{H}(9)$ & $4 e$ & 0.6085 & -0.0211 & -0.0557 & 0.076 \\
$\mathrm{H}(11 \mathrm{~A})$ & $4 e$ & 0.8009 & 0.3045 & -0.0622 & 0.082 \\
$\mathrm{H}(11 \mathrm{~B})$ & $4 e$ & 0.8049 & 0.2290 & -0.1180 & 0.082 \\
$\mathrm{H}(12 \mathrm{~A})$ & $4 e$ & 0.9877 & 0.3014 & -0.1638 & 0.130 \\
$\mathrm{H}(12 \mathrm{~B})$ & $4 e$ & 0.9592 & 0.3787 & -0.1152 & 0.130 \\
$\mathrm{H}(14)$ & $4 e$ & 0.7665 & 0.4736 & -0.1718 & 0.115 \\
\hline
\end{tabular}

* Correspondence author (e-mail: esobarzo@icaro.dic.uchile.cl) 
Table 2. Continued.

\begin{tabular}{llllll}
\hline Atom & Site & $x$ & $y$ & $z$ & $U_{\text {iso }}$ \\
\hline $\mathrm{H}(15)$ & $4 e$ & 0.5825 & 0.5106 & -0.2733 & 0.130 \\
$\mathrm{H}(18)$ & $4 e$ & 0.7895 & 0.2520 & -0.2699 & 0.101 \\
$\mathrm{H}(20 \mathrm{~A})$ & $4 e$ & 0.7484 & 0.1983 & -0.3884 & 0.254 \\
$\mathrm{H}(20 \mathrm{~B})$ & $4 e$ & 0.6032 & 0.1662 & -0.4404 & 0.254 \\
$\mathrm{H}(20 \mathrm{C})$ & $4 e$ & 0.6263 & 0.1640 & -0.3504 & 0.254 \\
$\mathrm{H}(22 \mathrm{~A})$ & $4 e$ & 0.3777 & 0.5114 & -0.3503 & 0.403 \\
$\mathrm{H}(22 \mathrm{~B})$ & $4 e$ & 0.3100 & 0.4985 & -0.4382 & 0.403 \\
$\mathrm{H}(22 \mathrm{C})$ & $4 e$ & 0.4589 & 0.5439 & -0.4121 & 0.403 \\
$\mathrm{H}(23 \mathrm{~A})$ & $4 e$ & 0.7583 & 0.2476 & 0.1194 & 0.071 \\
$\mathrm{H}(23 \mathrm{~B})$ & $4 e$ & 0.8947 & 0.2857 & 0.0966 & 0.071 \\
$\mathrm{H}(24 \mathrm{~A})$ & $4 e$ & 0.9026 & 0.1597 & 0.2090 & 0.075 \\
& & & & & \\
\hline
\end{tabular}

Table 2. Continued.

\begin{tabular}{llllll}
\hline Atom & Site & $x$ & $y$ & $z$ & $U_{\text {iso }}$ \\
\hline $\mathrm{H}(24 \mathrm{~B})$ & $4 e$ & 1.0412 & 0.1906 & 0.1834 & 0.075 \\
$\mathrm{H}(26)$ & $4 e$ & 0.8335 & 0.2296 & 0.3138 & 0.074 \\
$\mathrm{H}(29)$ & $4 e$ & 1.1285 & 0.4528 & 0.2948 & 0.076 \\
$\mathrm{H}(30)$ & $4 e$ & 1.1003 & 0.3472 & 0.2051 & 0.072 \\
$\mathrm{H}(32 \mathrm{~A})$ & $4 e$ & 1.2072 & 0.5047 & 0.4181 & 0.165 \\
$\mathrm{H}(32 \mathrm{~B})$ & $4 e$ & 1.1168 & 0.5641 & 0.4591 & 0.165 \\
$\mathrm{H}(32 \mathrm{C})$ & $4 e$ & 1.0849 & 0.5603 & 0.3689 & 0.165 \\
$\mathrm{H}(34 \mathrm{~A})$ & $4 e$ & 0.6850 & 0.2610 & 0.3950 & 0.197 \\
$\mathrm{H}(34 \mathrm{~B})$ & $4 e$ & 0.7282 & 0.2779 & 0.4840 & 0.197 \\
$\mathrm{H}(34 \mathrm{C})$ & $4 e$ & 0.8251 & 0.2206 & 0.4450 & 0.197 \\
$\mathrm{H}(10 \mathrm{~B})$ & $4 e$ & $1.025(4)$ & $0.282(2)$ & $0.003(2)$ & $0.13(1)$
\end{tabular}

Table 3. Atomic coordinates and displacement parameters (in $\AA^{2}$ ).

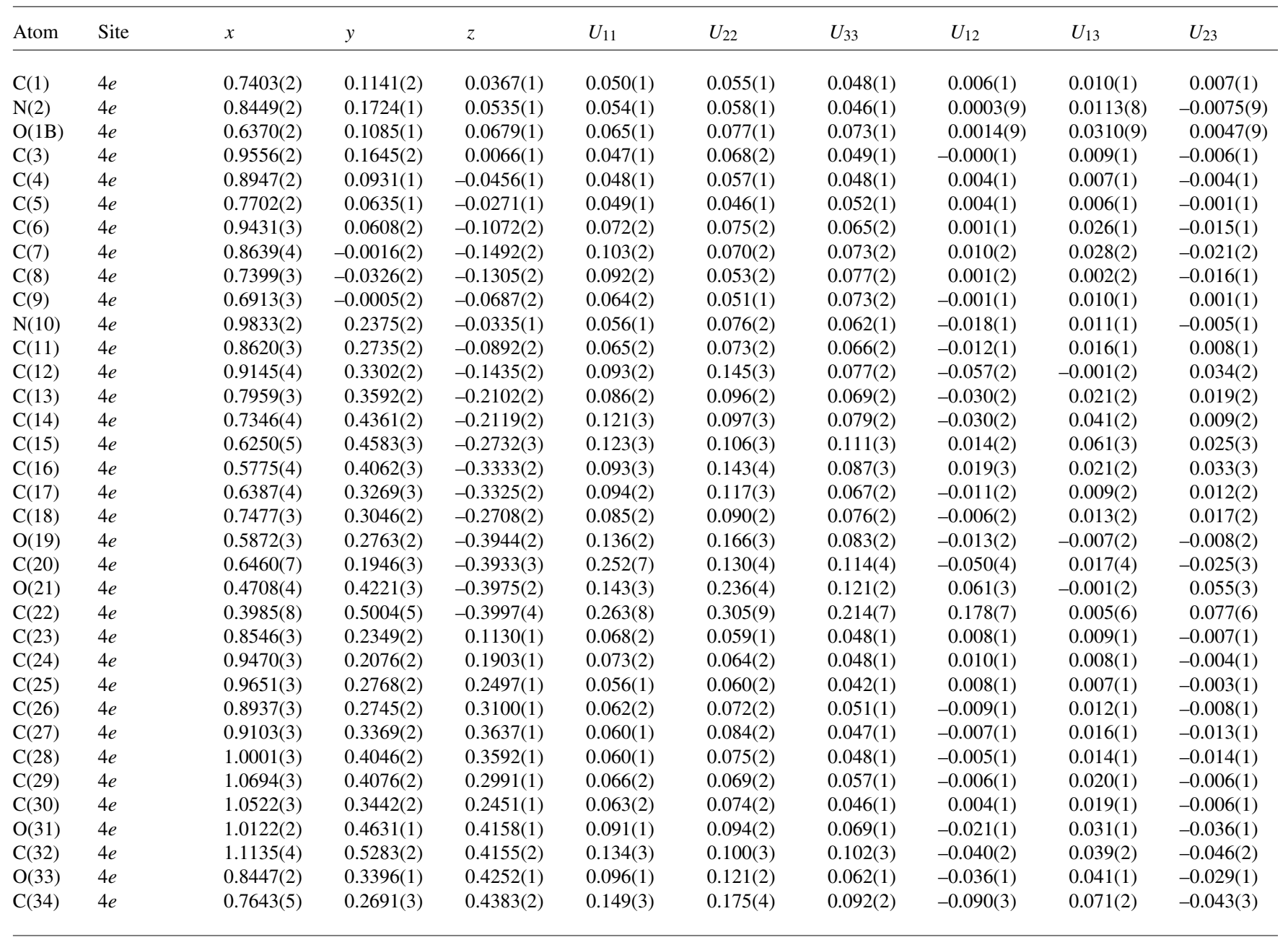

Acknowledgments. E. S.-S. thanks Fundación Andes for a doctoral fellowship. This work was funded in part by FONDECYT Grant No 2010056. Intensity measurements were performed at the Unidade de Raios X, RIAIDT, University of Santiago de Compostela, Spain.

\section{References}

1. Altomare, A.; Burla, M. C.; Camalli, M.; Carrozzini, B.; Cascarano, G. L.; Giacovazzo, C.; Guagliardi, A.; Moliterni, A. G. G.; Polidori, G.; Rizzi, R.: Sir97: A new tool for crystal structure determination and refinement. J. Appl. Crystallogr. 32 (1999) 115-119.

2. Sheldrick, G. M.: SHELXL-97. Program for the Refinement of Crystal Structures. University of Göttingen, Germany 1997.
3. Farrugia, L. J.: ORTEP-3 for Windows - a version of ORTEP-III with Graphical User Interface (GUI). J. Appl. Crystallogr. 30 (1997) 565.

4. Farrugia, L. J.: WinGX - Version 1.63.02. An integrated System of Windows Programs for the Solution, Refinement and Analysis of Single Crystals X-Ray Diffraction Data. J. Appl. Crystallogr. 32 (1999) 837-838. 